\title{
Führung und Machtmissbrauch
}

\section{Ein Erklärungsansatz zum Phänomen undemokratischer Führungsstrukturen in der Arbeitswelt}

Frank Relke

\begin{abstract}
Kernaussagen
Es gibt in der Arbeitswelt einen Widerspruch zwischen propagierten flachen Hierarchien und erlebtem undemokratischen Führungsverhalten. Machtmissbrauch wird vielerorts zum konstitutiven Führungsbestandteil, wobei die Machtinhaber ihr autoritäres Verhalten nicht reflektieren können. Das in diesem Zusammenhang vorkommende Ignorieren von Gesetzen ist nur eine von vielen Formen des Machtmissbrauchs. Der zunehmend zu beobachtende Rückgriff auf scheinbar alternativlose Entscheidungen ist ein Indiz für den Missbrauch der Macht. Undemokratisches Führungsverhalten entsteht aus der Angst heraus, eine erworbene Machtposition zu verlieren.
\end{abstract}

\section{Einleitung}

Seit geraumer Zeit gehören Schlagworte wie „flache Hierarchien“ und „kooperativer Führungsstil“ zu Profilbeschreibungen bei Stellenausschreibungen und Unternehmensleitbildern. Gleichzeitig häufen sich bei Personal- und Betriebsräten die Beschwerden über undemokratisches Führungsverhalten von Vorgesetzten. Ebenso sind in Fachzeitschriften wie in den Berufsressorts der überregionalen Tageszeitungen gehäuft Beiträge über konfliktverschärfendes Führungsverhalten von Vorgesetzten sowie Ratschläge für Führungspersonen zu lesen, wobei häufig mit Begriffen gearbeitet wird, die eher dem Fachgebiet der politischen Ordnungssysteme zuordenbar erscheinen. $\mathrm{Zu}$ nennen sind Formulierungen wie diktatorischer Führungsstil, monarchische oder feudalistische Strukturen. Wie kommt es zu diesem Widerspruch? Empirisch belastbare Daten bezüglich der Cheftypologien in Verwaltung und freier Wirtschaft liegen nicht vor und dürften auch nur mit einer großen Dunkelziffer zu erheben sein. Es bleibt bei der Feststellung des Phänomens der gehäuften Berichterstattung. Die Hypothese, nach der hierarchisch geführte Dienststellen und Betriebe grundsätzlich gefährdet sind, zu kleinen „Diktaturen“ zu werden, vermag der Verfasser dieses Beitrages nicht aufzustellen. Vielmehr soll der Blick auf das Phänomen gelenkt und zur weiteren Auseinandersetzung angeregt werden.

In politischen Ordnungssystemen geht es um Hierarchien und um Formen der Machtausübung. Macht kann durch Wahlen übertragen, durch einen Umsturz übernommen oder durch Erbfolge weitergegeben werden. Dass Vorgesetzte für ihre Mitarbeiter auch unbequeme Entscheidungen treffen müssen, ist ebenso unstrittig wie die Feststellung, dass die Arbeitsverhältnisse in der freien Wirtschaft wie im öffentlichen Dienst auch im 21. Jahrhundert grundsätzlich hierarchisch geprägt sind. Vorgesetzte können eher über charismatische oder eher über cholerische Persönlichkeitsstrukturen verfügen, was sich auf den Führungsstil und das Betriebsklima auswirken kann. Es ist jedoch ein Unterschied, ob ein Vorgesetzter durch seine Persönlichkeit führt, oder ob ein strukturelles Führungsproblem vorliegt. Um das Problemfeld greifbar werden zu lassen, wird das Beispiel des schulischen Dienststellenverhältnisses gewählt, da bei dieser Sonderform des beamtenrechtlichen Beschäftigungsverhältnisses durch Mitbestimmungsorgane wie Gesamt- und Schulkonferenzen eine Parallelität zwischen demokratischen und hierarchischen Elementen vorhanden ist. ${ }^{1}$ Vor diesem Horizont kann der Blick auf andere Hierarchieformen in der Verwaltung und in der freien Wirtschaft gerichtet werden. Angeregt durch Seminargespräche mit berufstätigen Studenten der Betriebswirtschaft und durch den Austausch mit Personalräten verschiedener Dienststellen ist der Verfasser zu der Hypothese gelangt, dass in vielen Dienststellen allenfalls pseudodemokratische Strukturen herrschen, wobei sich die Beschäftigten und die Führungsebene in der Gesamtheit dieser undemokratischen Strukturen nicht bewusst sind.

\section{Macht}

Eine neue Führungskraft wird mit der Intention den Dienst antreten, die Dienststelle modern und gut zu führen. Überholte Strukturen sollen aufgebrochen und der Teamgedanke gefördert werden. Wenn sich später herausstellen sollte, dass die Dienststelle einen „Diktator“ erhalten hat, muss es nicht sein, dass sich diese Person im Vorfeld ihres Führungsstils bewusst war. Auch diese Person kann ein von guten Werten geleiteter Mensch sein (Fisher, Ury \& Patton, 2009, S. 43 ff.). Aufgrund der Persönlichkeitsstruktur, die in der Regel mehrere unterschiedlich stark ausgeprägte Wertmuster enthält, oder aufgrund des hierarchisch vorgegebenen Organisationssystems wird sich bei dieser fiktiven Führungskraft das Verhaltensmuster des Machtmissbrauchs prozessual durchsetzen (Kernberg, 2000, S. 165 ff.). Am Ende des Prozesses werden andere Meinungen als die der Dienststellenleitung nicht mehr geduldet. Es kann so weit kommen, dass die Macht der Führungs-

1 Sofern in diesem Beitrag authentische Beispiele verwendet werden, wurden sie soweit abgeändert, dass keine Rückschlüsse auf die beteiligten Personen gezogen werden können. Mögliche Parallelen zu realen Ereignissen sind rein zufällig. 
ebene durch das Vorenthalten von Informationen gegenüber Beschäftigten und durch andere Täuschungen gesichert wird. Der Machtmissbrauch wird zum konstitutiven Führungsbestandteil.

An dieser Stelle ist zunächst eine Begriffsbestimmung notwendig. In Anlehnung an Max Weber kann Macht nach Lothar Döhn als die Möglichkeit bezeichnet werden, die der Einzelne oder eine Gruppe hat, um den eigenen Willen innerhalb einer sozialen Beziehung durchzusetzen (siehe den Lexikonbeitrag von Döhn, in: Drechsler, Hilligen, Neumann, 1992, S. 466). Macht ist nach Döhn die allgemeinere Form einer sozialen Beziehung, die sich erst in ihrer jeweiligen gesellschaftlichen Verwirklichung als Herrschaft darstellt. Sie bestehe in der Möglichkeit, eigene Interessen in für andere verbindliche Normen zu transformieren (ibid., S. 466). Weiter wird Macht im Zusammenhang mit einer Übertragungsleistung ausgeübt und kann in hierarchischen Systemen existent sein, wenn die Entscheidungsmöglichkeiten des Gegenübers eingeschränkt werden. Dabei ist die Macht des Machtinhabers von den Möglichkeiten des Adressaten abhängig, da das verlangte Handeln von den Adressaten realisierbar sein muss (Luhmann, 2012, S. 16-19, 96). Die Entscheidungsmöglichkeiten des Gegenübers können eingeschränkt werden, indem sich der Machtinhaber mit Verweis auf seine hierarchische Position über Gesetze hinweg setzt. Seinem Gegenüber wird durch diesen Machtmissbrauch Partizipation verwehrt. Die missachteten Gesetze verlieren ihre Macht, wie im Beispiel des nächsten Abschnitts gezeigt werden soll.

\section{Die Ohnmacht der Gesetze}

Wenn das Ergebnis zu einer Personalratswahl aufgrund der gesetzlichen Einspruchsfrist über den Zeitraum der Wahl hinaus ausgehängt bleiben muss, wundern sich mitunter Mitarbeiter darüber, warum die Bekanntmachung noch nicht abgenommen wurde. Den Hinweis auf die Aushangfrist, das Kleingedruckte, nimmt kaum jemand wahr. Ein Vorgesetzter äußert unter den Mitarbeitern seinen Unmut über diesen veralteten Aushang und nimmt ihn ab. Er wird auf den gesunden Menschenverstand hinweisen und den Personalrat ob der schlampigen Arbeitsweise lächerlich erscheinen lassen. Ein kritischer Personalrat wird den Vorgesetzten auf den Vorfall ansprechen. Schließlich geht es um das Einhalten von Gesetzen, die in demokratischen Prozessen verabschiedet wurden. Das Ignorieren von Gesetzen ist eine Form der Machtdemonstration, die nach außen mit pragmatischem Handeln begründet wird.

Auch die scheinbare Legalisierung von Entscheidungen durch Abstimmungen kann das faktische Ignorieren von Gesetzen bedeuten. Dienststellenleiter bedienen sich gerne des Instruments der Abstimmung, um gewünschte Entscheidungen herbeizuführen. Bei schulischen Gesamtkonferenzen, bei denen offene Abstimmungen üblich sind, sind volkskammerwahlähnliche Abstimmungsergebnisse keine Seltenheit. Das Plenum folgt in der Regel dem Vorschlag der Dienststellenleitung. Stimmenthaltungen gelten bereits als mutiges Zeichen.
Über den Wunsch nach geheimen Abstimmungen rümpfen Plenum und Dienststellenleitung, wenn auch aus unterschiedlichen Gründen, häufig die Nase. Dort, wo Abstimmungen in einer Demokratie vorgesehen sind, wird durch formale Grenzen ein Rahmen gesetzt, innerhalb dessen die Abstimmungen legal sind. Dienststellenleiter lassen jedoch mittels Abstimmungen auch solche Fakten schaffen, die durch Erlasse oder Verordnungen geregelt sind.

Gemäß Friedrich August von Hayek ist das beschriebene Verhalten mit einem naiven Glauben an die Demokratie gleichzusetzen, da durch die Missachtung der gesetzlichen Regelungen zugunsten von Kollektiventscheidungen die Freiheit des Einzelnen gefährdet wird (Horn, 2012, S. 213 ff.). Bei anderen Entscheidungen hingegen verkünden Dienststellenleiter eine von ihnen getroffene Entscheidung als alternativlos, obwohl die Partizipation der Mitarbeiter vorgesehen wäre. $\mathrm{Zu}$ den alternativlosen Entscheidungen folgen Ausführungen im nächsten Abschnitt. An dieser Stelle genügt der Hinweis auf drohende Willkür, wobei den von den Dienststellenleitern gewünschten Entscheidungen Vorstellungen darüber zugrunde liegen, was für die Dienststelle gut ist. Die Autorität bedarf jedoch, auch wenn sie ihre Entscheidungen durch Abstimmungen legalisieren lassen will, einer Selbstbeschränkung durch das Recht. Eine Dienststelle ist ein atmendes Gebilde, das sich im Rahmen der gesetzlichen Vorgaben entwickelt. Wenn dieses Atmen unterbunden wird, wird aus der Herrschaft des Rechts mithilfe von geschickten Inszenierungen eine Ohnmacht der Gesetze (Horn, 2012, S. 213 ff.).

Elemente der Inszenierung können sein:

- Sitzordnung dem Anlass entsprechend festlegen.

- Wir-Gefühl ansprechen (an Verantwortung appellieren, Zukunft im Blick haben).

n Außergewöhnliche Rahmenbedingungen erwähnen (bewusst gewählte Zeit, Sitzordnung).

- Dringlichkeit der Entscheidung und Alternativlosigkeit betonen.

\section{Alternativlose Entscheidungen}

Wenn Angehörigen einer Dienststelle Entscheidungen, an denen sie grundsätzlich Mitbestimmungsrechte haben, von der Leitungsebene mit der Begründung, die Entscheidung sei ohnehin alternativlos, abgenommen werden, ist dies Machtmissbrauch. Gemäß der Rhetorik der Leitungsebene wird die Entscheidung zum Wohl der Mitarbeiter getroffen worden sein. In einem insgesamt demokratischen System, dessen Atmosphäre von gegenseitigem Vertrauen geprägt ist, ist es nicht notwendig, einer Entscheidung das Prädikat alternativlos zu verleihen. Der Adressat kann sich sicher sein, dass der Sender die Entscheidung reiflich überlegt hat. Es stellt keinen großen Verlust dar, wenn sich im Nachhinein herausstellt, dass eine andere Entscheidung die günstigere gewesen wäre. Das Wissen um die Qualität des Lernens aus Fehlern sowie das Eingestehen der Fehlentscheidung machen den Beteiligten das Vergeben leicht. 
Im Gegensatz dazu steht das von Misstrauen geprägte System. An der Rhetorik der alternativlosen Entscheidungsbegründung wird dies deutlich. Mit der Begründung der Alternativlosigkeit werden die Gegenspieler mundtot gemacht (Hoffmann, 2012). Es gibt nichts mehr abzuwägen. Ein Diskurs bzw. ein Wettkampf um den besten Weg ist nicht mehr notwendig. Es kann von einer Gleichschaltung der Meinung gesprochen werden, da die für ein demokratisches System konstitutive Meinungsvielfalt nicht artikuliert werden darf. In einem undemokratischen System sind kritisch innovative Worte nicht gefragt, da das System nicht atmen soll.

Weil es keine alternativlosen Lösungen gibt, können die angeblich alternativlosen Entscheidungen, die stets, vom Führungsteam gemeinsam getroffen' wurden, nur mit oberflächlichen Floskeln wie ,wohl überlegt', , alle Eventualitäten bereits im Vorfeld abgewogen', ,gewissenhaft durchdacht' und mit Halbwahrheiten begründet werden. Mit Halbwahrheiten ist noch keine Lüge verbunden. Eine Lüge könnte entlarvt werden. Die Konfrontation mit einer Lüge könnte für den Lügner nicht nur peinlich werden; sie kann die wahren Absichten aufdecken. Mit Halbwahrheiten hingegen ist der Versuch verbunden, sich die Dinge zurechtzubiegen; die Grenzen zur Lüge werden unklar (Frey, Schnabel, 2012; Wilfling, 2011, S. 132, S. 294). Menschen, die Entscheidungen mit der Alternativlosigkeit begründen, können als durchsetzungsstark oder aber auch als gefühlskalt empfunden werden. Dabei wird verdrängt, dass auch diese Menschen von Gefühlen geleitet werden. Ein bedeutendes Gefühl ist in diesem Zusammenhang die Angst.

\section{Angst und Macht}

Jedes Gefühl hat eine Ursache und ist mit dem Verhalten anderer Personen verbunden. Angst, Ärger, Trauer und Freude gelten als die vier Ursprungsgefühle. Sie stehen in einem zeitlichen Bezug und verfügen über einen Auslöser und einen Handlungsimpuls (Risto, 2005, S. 114 ff.). Das Handeln von Menschen in Machtpositionen wird häufig von dem Ursprungsgefühl der Angst geleitet. Diesen Zusammenhang haben Dieter Frey und Albrecht Schnabel im Kontext der Wulff-Affäre in einem Gastbeitrag hergeleitet (Frey, Schnabel, 2012; Hauser 2000, S. 154 ff.). Der Auslöser ist die starke Befürchtung, dass sich ein Ist- Zustand verschlimmert. Die Zeitperspektive liegt in der Zukunft, der Handlungsimpuls ist das Ergreifen von Schutzmaßnahmen (Risto, 2005, S. 114 ff.).

Führungskräfte können unter der Angst leiden, dass die erarbeitete Position verloren gehen kann. Wer im Zentrum der Macht ist, umgibt sich mit einer Wagenburg aus konzentrischen Ringen. Diese besteht aus Günstlingen unterschiedlicher Hierarchieebenen, die den obersten Machtinhaber bestätigen und mit Informationen versorgen (Koschorke, 2008, S. 68 ff.) Im Sinne dieses Bildes sind die Schulleiter vom Schulleitungsteam und von schulleitungsnahen Kollegen umgeben. Die Mitglieder der Wagenburg sind dem Schulleiter zu Dank verpflichtet, da dieser ihnen dabei geholfen hat, auf der Karriereleiter einen Schritt nach oben zu gelangen.
Bei einem Machtinhaber können durch die Machtfülle Omnipotenzgefühle und eine Arroganz der Macht entstehen. Der Amtsinhaber, der von dieser Persönlichkeitsdisposition geprägt ist, wird nicht nur den Kontakt zur Basis verlieren, sondern sich auch im privaten Umfeld im Dunstkreis seinesgleichen aufhalten. Die Mitgliedschaft im Schützenverein dürfte allenfalls passiv bestehen und als Rudiment aus einer längst vergangenen persönlichen Epoche zu verstehen sein. Die Folge ist Realitätsverlust aufgrund von fehlendem Widerspruch und kritischem Feedback. Wenn es zu kritischen Äußerungen bezüglich der getroffenen Entscheidungen kommt, werden diese als verletzend empfunden. Da weder interne noch externe Korrekturmechanismen greifen können, wird der von Angst geleitete Machtmensch mit Drohungen reagieren, während bei einer selbstreflexiven Persönlichkeit die internen Kontrollmechanismen greifen würden (Frey, Schnabel, 2012). Eingangs wurde mit dem Verweis auf Luhmann hergeleitet, dass die Macht des Machtinhabers von den Möglichkeiten des Adressaten abhängig ist. Von den Möglichkeiten des Adressaten war in diesem Beitrag bislang nur am Rande die Rede, was im nächsten Abschnitt nachgeholt werden soll.

\section{Die Unterstützer des Systems}

Es gibt bewusste und unbewusste Unterstützer eines Systems. Bewusste Unterstützer erhoffen sich von der Unterstützung der Macht Vorteile, während ein unbewusster Unterstützer ein Medium ist. Er transportiert verbale Botschaften oder wirkt durch sein linientreues Handeln unterstützend. Macht braucht Masse. Macht braucht Freunde. Diese kleinen Sätze beschreiben ein konstitutives Machtmoment. Freunde werden nicht nur für die Aufrechterhaltung von Beziehungen benötigt, sondern auch um Brücken zum anderen Lager zu errichten.

Die „böse Macht“ erfindet selten etwas Neues. Vielmehr werden Teile bestehender Ordnung auf den Kopf gestellt. Bei der Säule der Freundschaft wird Vertrauen in Misstrauen umgemünzt. Eine Dreiecksbeziehung wird hergestellt: Der Abteilungsleiter (A) hat eine kollegial-freundschaftliche Beziehung zu einem Mitarbeiter (M) aus der Nicht-Führungsebene aufgebaut. Dieser wird vorgeschickt, um einen Mitarbeiter der Opposition (O) mit ungeliebten Aufgaben zu beauftragen. M eignet sich dazu, da er in einem neutralen Verhältnis zu O steht. Es ist entscheidend, wer eine Botschaft überbringt. Wenn $\mathrm{M}$ die Botschaft überbringt, ist es für O lediglich eine unangenehme Botschaft. Wenn jedoch A die Botschaft übermittelt, handelt es sich um eine Anweisung des übergeordneten Gegners, die eine Gegenreaktion hervorrufen würde. Ob M sich dieses Freundschaftsverhältnisses bewusst ist, kann von Fall zu Fall unterschiedlich sein.

Eine weitere tragende Säule des Systems ist der Durchschnittsmitarbeiter, der weder in Konferenzen noch bei Betriebsfeiern auffällt. Der Staatsdiener möchte in Ruhe arbeiten und nicht mit unangenehmen Situationen konfrontiert werden. Beispiel: Für eine Evaluation wird unter den Lehrern, Eltern und Schülern eine Onlinebefragung durchgeführt. Kurz 
vor Ablauf der Frist ist absehbar, dass erst ein geringer Prozentsatz an der Befragung teilgenommen hat. Zuvor sagte Herr Linientreu den Schülern, dass die Teilnahme freiwillig sei. Nachdem die Schulleitung nachgefragt hat, welche Klassen bislang noch nicht an der Befragung teilgenommen haben, lässt Herr Linientreu die Fragebögen von den Schülern in der Schule ausfüllen. Das Ausfüllen des Fragebogens sei Pflicht.

Der Oxford-Professor Paul Collier äußert sich in einem Interview zur Finanzkrise, dass eine Demokratie nur so gut sei wie das Wissen der Bürger, auf denen sie aufbaue. Das System funktioniere nur, wenn eine kritische Masse der Bürger die Probleme verstehe (Marc Goergen: Demokratie-hi-hi-hi. Sind Wahlen Luxus? Sind Diktaturen ökonomisch effizienter?, in: Stern 24/2012, S. 107). Auf den hier behandelten Kontext bezogen bedeutet dies, dass Machtmissbrauch in der Arbeitswelt so reibungslos funktioniert, weil die in ihr arbeitenden Individuen über kein hinreichendes systemrelevantes Wissen verfügen und die Probleme nicht sehen. Es ist zu klären, ob den Beschäftigten Informationen bewusst vorenthalten werden oder ob sie die Informationen aufgrund eines grundsätzlichen Desinteresses nicht suchen.

Dies wirft auch Fragen nach der inneren Grundhaltung dieser Personen auf. Inwieweit identifizieren sie sich mit ihrem Beruf? Leben sie ihre Berufsidentität als Schauspieler, der jeden Tag auf der Bühne steht und süchtig nach Applaus ist? Oder leben sie ihre Berufsidentität als schizophrene Saubermänner, die oberflächlich Demokratie lehren und dem Dienstherren aus Opportunitätsgründen folgen? Festzuhalten bleibt, dass beide Seiten, der Machtunterworfene sowie der Machtausübende, stets über Alternativen verfügen (Luhmann, 2012, S. $29 \mathrm{ff}$.$) .$

\section{Schluss}

Im Rahmen dieses Beitrags kann die Frage, ob der von vielen Führungskräften praktizierte Machtmissbrauch eine Folge der Struktur oder der Persönlichkeit ist, nicht abschließend geklärt werden. Ein monokausaler Erklärungsansatz erscheint für diese komplexe Thematik unzureichend, zumal diesbezüglich in der wissenschaftlichen Diskussion eine Ambivalenz zu beobachten ist: Einerseits kommen Untersuchungen bislang zu dem Ergebnis, dass Führungskräfte tendenziell als gewissenhaft, offen für Innovationen und emotional stabil bezeichnet werden können. Auf der anderen Seite belegen Forschungsergebnisse, dass Personen mit dem Erlangen von Führungspositionen ihr Verhalten ändern. Sie werden impulsiver und reflektieren ihr Handeln weniger (Myriam Bechtoldt im Interview mit Harald Freiberger, in: Süddeutsche Zeitung, 3.1.2013, S. 18; Kernberg, 2000).

\section{Literatur}

Drechsler, H., Hilligen, W. \& Neumann, F. (Hrsg.) (1992). Gesellschaft und Staat: Lexikon der Politik (8. Auflage). München: Franz Vahlen.

Fisher, R., Ury, W., \& Patton, B. (Hrsg.) (2009). Das Harvard-Konzept: Der Klassiker der Verhandlungstechnik (23. Auflage). Frankfurt: Campus.
Freiberger, H. (2013). „Der Mächtige bleibt ungerührt“. Süddeutsche Zeitung, 3.1.2013, 18.

Frey, D. \& Schnabel, A. (2012). Die Wulffs sind unter uns. Süddeutsche Zeitung, 20.1.2012, 18.

Goergen, M. (2012). Demokratie-hi-hi-hi. Sind Wahlen Luxus? Sind Diktaturen ökonomisch effizienter? Interview mit Paul Collier. Stern 24/2012, 107.

Hauser, M. (2000). Charismatische Führung. Wiesbaden: Deutscher Universitäts-Verlag.

Hoffmann, C. (2012). Widerrede erwünscht [Electronic version]. Frankfurter Allgemeine Zeitung, 2.4.2012.

Horn, K. (2012). Was kann der Staat, was darf die Wirtschaft? In W. Plumpe (Hrsg.), Der Staat und die Ordnung der Wirtschaft: Vom Kaiserreich bis zur Berliner Republik (205-226). Stuttgart: Franz Steiner.

Kernberg, O. (2000). Ideologie, Konflikt und Führung. Stuttgart: KlettCotta.

Koschorke, A. (2008). Das Volk als Gerücht: Zur Labilität souveräner Herrschaft im Barockdrama. In J. Brokoff (Hrsg.). Die Kommunikation der Gerüchte (68-78). Göttingen: Wallstein.

Luhmann, N. (2012). Macht im System. Berlin: Suhrkamp.

Risto, K. (2005). Konflikte lösen mit System: Mediation mit Methoden der Transaktionsanalyse (2. Auflage). Paderborn: Junfermann.

Wilfling, J. (2011). Abgründe: Wenn aus Menschen Mörder werden (2. Auflage). München: Heyne.

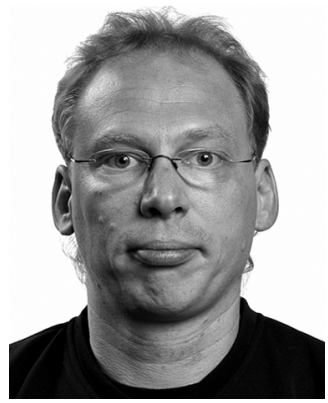

Dr. Frank Relke ist Lehrer und Honorardozent für die Fächer kooperative Konfliktlösung und Professionalisierung beruflichen Handelns. 2009 schloss er seine Dissertation zum Thema „Politische Bildung und Evangelische Kirche" an der Universität Kassel ab. Email: frankrelke@me.com 